\title{
¿Cuál es el conocimiento básico que los profesores necesitan para ser más efectivos en sus clases? El caso del concepto Reacción química.
}

\author{
Elizabeth Nieto Calleja* \\ Andoni Garritz* \\ Flor Reyes-Cárdenas**
}

Artículo recibido: 25-8-2007 y aprobado: 12-10-2007

What is the knowledge base that teachers need
to be more effective in their classes? The case
of the concept of 'chemical reaction'.

Resumen: Shulman introdujo en 1987 el concepto de conocimiento básico de un profesor concebido como un producto de la investigación: "Los hallazgos de la investigación sobre la enseñanza efectiva son típicamente considerados como parte legítima de un conocimiento básico". Para Shulman dar una clase efectiva implica poseer unas habilidades básicas, conocimiento del contenido y habilidades pedagógicas generales, pero concibe estos dos últimos elementos amalgamados en lo que denominó "conocimiento pedagógico del contenido" (CPC). En este artículo se documenta y discute el CPC de cinco profesores experimentados en su curso de Química General Universitaria sobre el concepto de Reacción Química. Hemos seguido la metodología de la Representación del Contenido (ReCo) y de los Repertorios de experiencia Profesional y Pedagógica (RePyPs) de Loughran et ál. Como muestra final de lo que puede obtenerse con dichos documentos hemos propuesto en las conclusiones un decálogo de puntos importantes a considerar cuando se pretenda dar una clase efectiva sobre este tema.

Palabras Clave: Conocimiento base, conocimiento pedagógico del contenido, representación del córitenido, repertorios de experiencia profesional y pedagógica, reacción química, educación superior.

Abstract: In 1987, Shulman introduced the concept of knowledge base of a teacher conceived as a result of research:"The findings of research on effective teaching are typically deemed as a legitimate part of a knowledge base". Teaching requires basic skills, content knowledge and general pedagogical skills, but Shulman conceive the last two elements amalgamated in what he defined as "pedagogical content knowledge" (PCK). In this paper the PCK of five General Chemistry teachers on the topic "Chemical reaction" is documented and discussed by using the methodology of the Content Representation (CoRe) and the Pedagogical and Professional experience Repertories (PaP-eRs) proposed by Loughran et ál. As a final sample of what can be concluded with these types of document a Decalogue of important points to consider when an effective class on this topic is pretended.

Keywords: Knowledge base, pedagogical content knowledge, content representation, chemical reaction, higher education.

\footnotetext{
* Profesores de la Facultad de Química, Universidad Nacional autónoma de México, UNAM. liz@servidor.unam. mxy andoni@servidor.unam.mx

**Especialista química en el proyecto Enciclomedia, ILCE. florreyes@gmail.com

1 El contenido de este artículo fue presentado por Elizabeth Nieto en el Seminario de Investigación Educativa de la Facultad de Química de la Universidad Nacional Autónoma de México (ver la URL de Metl, “Papeles del SIE": http://depa.pquim.unam.mx/sie/publicaciones.htm).
} 


\section{Introducción}

El reto actual en la clase de Ciencias ya dejó de ser transmitir información, ahora hay que enseñar a utilizarla, a establecer relaciones entre enunciados aparentemente dispares y, muy especialmente, a comunicar nuestras ideas y debatirlas con las expresadas por los demás.

Shulman (1987) nos habla de la base de conocimientos del profesor como algo que vale la pena averiguar y discutir desde sus fuentes, para formar nuevos profesores, por ejemplo, este autor explora el proceso de razonamiento pedagógico y de acción en el cual tal conocimiento del profesor es empleado. Nos dice, "El profesor puede transformar el entendimiento, las habilidades de desempeño o las actitudes y valores deseados en representaciones y acciones pedagógicas. Éstas son formas de hablar, mostrar, promulgar o representar ideas de tal forma que lo desconocido pueda volverse conocido, para que aquellos sin entendimiento puedan comprender y discernir, y para volver aptos a los no calificados. Por ello, enseñar necesariamente empieza con la comprensión del profesor de qué se debe aprender y cómo debe ser enseñado". Después de más de quince años de haberse propuesto los conceptos de base de conocimiento y de conocimiento pedagógico del contenido por Shulman (1986), de Jong, Veal y van Driel (2002) han escrito un capítulo titulado Exploring chemistry teachers' knowledge base en el que recopilan los trabajos que, de alguna forma, han tenido que ver con el desarrollo de esta rama de la investigación sobre la enseñanza de la Química.
Desde hace más de dos décadas, numerosos investigadores de la enseñanza de las ciencias se han dado a la tarea de reflexionar acerca de la acción de los docentes en el aula, como dicen Pozo y Gómez Crespo (1997), al indagar los aprendizajes implícitos de los profesores en relación con su tarea y las formas en que los hacen explícitos. Estos autores agregan al final de su libro: "La probabilidad de éxito será mayor cuando las decisiones [sobre fijar las metas y las actividades de enseñanza y evaluación a desarrollar] sean coherentes con los supuestos de ese profesor o grupo de profesores sobre la naturaleza del conocimiento científico y su aprendizaje[...] aprender a enseñar Ciencia requiere de los profesores un cambio conceptual, procedimental y actitudinal no menos complejo que el que exige a los alumnos el propio aprendizaje de la Ciencia”.

En este trabajo pretendemos documentar el conocimiento base de cinco profesores de nivel universitario sobre el concepto de 'reacción química', complementando lo que ya informaron dos de los autores (Reyes y Garritz, 2006).

\section{Marco teórico}

Los profesores deben conocer bien cuál es el objetivo de su enseñanza, qué es lo que resultará fácil o difícil de aprender para sus alumnos, cuáles son las concepciones alternativas (CA) más comunes en ellos, y cómo organizar, secuenciar, presentar y evaluar el contenido para abastecer los diversos intereses y capacidades de sus alumnos. Todo este conocimiento se resume en el Conocimiento Pedagógico del Contenido, CPC (Garritz y Trinidad-Velasco, 2004; 2006). 
El CPC se refiere al producto de la interacción entre el contenido temático de la materia y la Pedagogía, y al tipo de conocimiento que los profesores de ciencias deben poseer, ya que no sólo tienen que conocer y entender el tema científico, sino también cómo enseñar ese contenido de forma efectiva (Barnett y Hodson, 2001; Garritz y Valdés, 2007).

El propósito de este trabajo es presentar los resultados de utilizar la metodología aplicada para representar el CPC de los profesores, utilizando la Representación del Contenido (ReCo) y los Repertorios de experiencia Profesional y Pedagógica (Re PyPs) informados por un grupo australiano de investigación educativa: Loughran, Mulhall y Berry (2004). Hemos seguido la metodología propuesta por estos autores para obtener las ReCos de cinco profesores de nivel universitario sobre reacción química y los Re-PyPs de dos de ellos (ver el artículo de Loughran et ál., 2001, donde se les llama PaP-eRs, por sus siglas en Inglés, o Inventarios, como los llaman Raviolo y Garritz, 2005).

La ReCo establece y discute el entendimiento de los profesores de ciencias sobre aspectos particulares del CPC, empezando por declarar las ideas centrales alrededor del tema específico; la importancia de que los alumnos las adquieran; el conocimiento de las CA de los alumnos; los puntos conocidos de confusión en ellos; el establecimiento de una secuencia efectiva; las estrategias didácticas y las formas de evaluarlas. Los inventarios muestran los aspectos de la enseñanza y aprendizaje de un tema en particular.

\section{Metodología}

Se seleccionaron cinco profesores destacados del nivel de licenciatura, con una antigüedad promedio de treinta años, que hubieran impartido al menos durante diez años la asignatura Química General, que se caracterizaran por involucrarse en un tipo de enseñanza centrado en los estudiantes, o sea, uno no tradicional, y que contaran con libros y otros materiales impresos relacionados con la enseñanza.

Cada uno de los cinco profesores del nivel universitario fue entrevistado, con el propósito de aclarar lo que debería considerarse como 'ideas centrales' en el cuestionario (ver Tabla 1) que nos proponen Loughran, Mulhall y Berry (2004).

Al respecto se les aclaró que las 'ideas centrales' son los tópicos que forman parte del conocimiento disciplinario en los que acostumbran dividir la enseñanza del concepto de Reacción Química y que constituyen el corazón central del tema.

Se trata de que en ese conjunto de ideas estén reflejadas aquellas que son las más importantes del tema a impartir, o de sus precedentes, y que constituyan un referente importante de la manera como dividen en porciones sus clases. 
Tabla 1. Base de la ReCo (Representación del contenido)

Base de las entrevistas.

¿Cuáles son las ideas científicas que se encuentran en el centro del tema Reacción química (RQ)? Es decir, seleccione entre tres a cinco ideas en las que acostumbre dividir la enseñanza del concepto de $\mathrm{RQ}$. Se trata de que en ese conjunto de ideas estén reflejadas las más importantes del tema a impartir, o de sus precedentes.

Para cada una de estas ideas responda las siguientes preguntas:

1. ¿Qué intenta que aprendan los alumnos alrededor de esta idea?

2. ¿Por qué es importante que los alumnos sepan esta idea?

3. ¿Qué más sabe respecto a esta idea? (y qué no incluye en sus explicaciones a sus alumnos).

4. ¿Cuáles son las dificultades o limitaciones relacionadas con la enseñanza de esta idea?

5. ¿Qué conocimientos acerca del pensamiento de los alumnos influyen en su enseñanza de esta idea?

6. ¿Qué otros factores influyen en su enseñanza de esta idea?

7. ¿Cuáles procedimientos de enseñanza emplea? (y las razones particulares de su uso con esta idea).

8. ¿Qué formas específicas de evaluación del entendimiento o de la confusión de los alumnos emplea alrededor de esta idea?

\section{Resultados}

Encontramos en ellos un gran abanico de ideas centrales citadas para la enseñanza de la RQ (ver Tabla 2). Vemos que algunas de ellas son tópicos demasiado específicos sobre el tema o sus antecedentes y que han sido informadas con demasiada brevedad, como por ejemplo la 'fórmula única para cada sustancia' o 'la Ley de Coulomb'.

Observamos que hay similitud y relativa homogeneidad en los cinco primeros renglones.

Tabla 2. Ideas centrales de los cinco profesores entrevistados

\begin{tabular}{|c|c|c|c|c|c|}
\hline Ideas centrales & Profesor 1 & Profesor 2 & Profesor 3 & Profesor 4 & Profesor 5 \\
\hline $\begin{array}{l}\text { Relaciones } \\
\text { cuantitativas de la } \\
\text { reacción química } \\
\text { y balanceo de } \\
\text { ecuaciones. }\end{array}$ & Balanceo & $\begin{array}{l}\text { Relaciones } \\
\text { cuantitativas del } \\
\text { cambio químico. }\end{array}$ & $\begin{array}{l}\text { Nomenclatura y } \\
\text { estequiometría }\end{array}$ & $\begin{array}{l}\text { Balanceo de } \\
\text { una ecuación } \\
\text { química. }\end{array}$ & $\begin{array}{l}\text { Relaciones en } \\
\text { masa y en cantidad } \\
\text { de sustancia, } \\
\text { reactivo limitante, } \\
\text { rendimiento, pureza } \\
\text { de los reactivos, etc. }\end{array}$ \\
\hline $\begin{array}{l}\text { Conservación de la } \\
\text { masa o de los átomos. }\end{array}$ & $\begin{array}{l}\text { Conservación } \\
\text { de núcleos } \\
\text { y electrones } \\
\text { (conservación de la } \\
\text { masa). }\end{array}$ & $\begin{array}{l}\text { Conservación de } \\
\text { la masa. } \\
\text { Conservación de } \\
\text { átomos. }\end{array}$ & & $\begin{array}{l}\text { Conservación } \\
\text { de la masa. } \\
\text { Conservación } \\
\text { de átomos. }\end{array}$ & $\begin{array}{l}\text { Conservación de la } \\
\text { masa. } \\
\text { Conservación de los } \\
\text { átomos. }\end{array}$ \\
\hline $\begin{array}{l}\text { Representación de las } \\
\text { reacciones. }\end{array}$ & & $\begin{array}{l}\text { Representación de } \\
\text { reacciones. }\end{array}$ & $\begin{array}{l}\text { Representación } \\
\text { simbólica de una } \\
\text { reacción. }\end{array}$ & $\begin{array}{l}\text { Una reacción } \\
\text { química real } \\
\text { se representa } \\
\text { por medio de } \\
\text { una ecuación } \\
\text { química. }\end{array}$ & $\begin{array}{l}\text { Representación de } \\
\text { reacciones. }\end{array}$ \\
\hline
\end{tabular}


Tecné, Episteme y Didaxis n.o 22,2007

\begin{tabular}{|c|c|c|c|c|c|}
\hline Ideas centrales & Profesor 1 & Profesor 2 & Profesor 3 & Profesor 4 & Profesor 5 \\
\hline $\begin{array}{l}\text { Formación de nuevas } \\
\text { sustancias. }\end{array}$ & & $\begin{array}{l}\text { Formación de } \\
\text { nuevas sustancias. }\end{array}$ & & $\begin{array}{l}\text { Formación } \\
\text { de nuevas } \\
\text { sustancias. }\end{array}$ & $\begin{array}{l}\text { Formación de } \\
\text { nuevas sustancias. }\end{array}$ \\
\hline $\begin{array}{l}\text { Tipos de reacciones } \\
\text { químicas. }\end{array}$ & & $\begin{array}{l}\text { Tipos de } \\
\text { reacciones } \\
\text { químicas. }\end{array}$ & $\begin{array}{l}\text { Clasificación de } \\
\text { las reacciones. }\end{array}$ & $\begin{array}{l}\text { Tipo de } \\
\text { reacciones } \\
\text { químicas. }\end{array}$ & \\
\hline $\begin{array}{l}\text { Naturaleza } \\
\text { corpuscular de las } \\
\text { sustancias. }\end{array}$ & $\begin{array}{l}\text { Naturaleza } \\
\text { corpuscular de las } \\
\text { sustancias. }\end{array}$ & $\begin{array}{l}\text { Modelo } \\
\text { corpuscular. }\end{array}$ & & $\begin{array}{l}\text { Modelo } \\
\text { corpuscular. }\end{array}$ & \\
\hline $\begin{array}{l}\text { Naturaleza eléctrica } \\
\text { de las partículas. }\end{array}$ & $\begin{array}{l}\text { Naturaleza } \\
\text { eléctrica de las } \\
\text { partículas. }\end{array}$ & & & & \\
\hline $\begin{array}{l}\text { Discontinuidad de la } \\
\text { materia. }\end{array}$ & $\begin{array}{l}\text { Discontinuidad de } \\
\text { la materia. }\end{array}$ & $\begin{array}{l}\text { Discontinuidad de } \\
\text { la materia. }\end{array}$ & & $\begin{array}{l}\text { Discontinuidad } \\
\text { de la materia. }\end{array}$ & $\begin{array}{l}\text { Discontinuidad de } \\
\text { la materia. }\end{array}$ \\
\hline $\begin{array}{l}\text { El concepto de } \\
\text { cantidad de sustancia } \\
\text { y su unidad el mol. }\end{array}$ & & $\begin{array}{l}\text { Concepto de } \\
\text { cantidad de } \\
\text { sustancia. }\end{array}$ & $\begin{array}{l}\text { Concepto de } \\
\text { mol. }\end{array}$ & $\begin{array}{l}\text { Concepto de } \\
\text { cantidad de } \\
\text { sustancia y su } \\
\text { unidad el mol. }\end{array}$ & $\begin{array}{l}\text { El concepto de } \\
\text { cantidad de } \\
\text { sustancia y su } \\
\text { unidad el mol. }\end{array}$ \\
\hline Ley de Coulomb. & Ley de Coulomb. & & & & \\
\hline $\begin{array}{l}\text { Conservación de la } \\
\text { energía. }\end{array}$ & $\begin{array}{l}\text { Conservación de la } \\
\text { energía. }\end{array}$ & & & & \\
\hline Tabla periódica. & & & Tabla periódica. & & \\
\hline $\begin{array}{l}\text { Enlace y equilibrio } \\
\text { químico. }\end{array}$ & & & $\begin{array}{l}\text { Enlace y } \\
\text { equilibrio } \\
\text { químico. }\end{array}$ & & \\
\hline $\begin{array}{l}\text { Concepto de } \\
\text { sustancia, átomo y } \\
\text { molécula. }\end{array}$ & $\begin{array}{l}\text { Concepto de } \\
\text { sustancia. }\end{array}$ & $\begin{array}{l}\text { Concepto de } \\
\text { sustancia. }\end{array}$ & & $\begin{array}{l}\text { Concepto de } \\
\text { sustancia, } \\
\text { átomo y } \\
\text { molécula. }\end{array}$ & $\begin{array}{l}\text { Concepto de } \\
\text { sustancia, átomo y } \\
\text { molécula. }\end{array}$ \\
\hline $\begin{array}{l}\text { Fórmula única para } \\
\text { cada sustancia. }\end{array}$ & & & & & $\begin{array}{l}\text { Fórmula única para } \\
\text { cada sustancia. }\end{array}$ \\
\hline
\end{tabular}

Tabla 3. Las cinco ideas centrales más comunes entre los cinco profesores y su comparación con las cinco ideas centrales informadas para profesores australianos (Mulhall, Berry y Loughran, 2003)

\begin{tabular}{ll}
\hline Investigación mexicana & Investigación australiana \\
\hline Formación de nuevas sustancias. & En una reacción química (una o más) nuevas sustancias se producen. \\
\hline Tipos de reacciones. & Existen patrones para muchas reacciones químicas. \\
\hline Representación de reacciones. & Las ecuaciones describen a los reactivos y productos en una reacción. \\
\hline $\begin{array}{l}\text { Relaciones cuantitativas de la reacción química } \\
\text { y balanceo. }\end{array}$ & \\
\hline Conservación de la masa o de los átomos. & Las sustancias químicas pueden ser representadas por fórmulas. \\
\hline & Los productos químicos orgánicos contienen carbón.
\end{tabular}

En los colegas australianos no aparecen ideas centrales sobre los aspectos submicroscópicos (en adelante usaremos "nanoscópicos", que está más de acuerdo con la medida real de los átomos y las moléculas), o acerca de la conservación de la masa.
Ahora revisaremos algunas expresiones interesantes incluidas en la ReCo de los profesores entrevistados y se comentarán de acuerdo con algunas recomendaciones didácticas que se han dado en artículos recientes. 
I. Con relación a la idea central de 'conservación de la materia', y la pregunta 2 del cuestionario de la Tabla 1, que se refiere a la importancia del tema para los estudiantes, transcribimos a continuación lo expresado por tres de los profesores:

La parte fundamental en este aspecto es que los estudiantes aprendan que durante una reacción química se forman nuevas sustancias cuyas propiedades son muy diferentes a las de aquellas de las que partieron, y que en ese proceso, la masa se mantiene constante si la reacción se lleva a cabo en un sistema cerrado.

La necesidad de dar nombre a los compuestos químicos. De introducir las leyes de la conservación de la masa en las reacciones químicas. Que comprendan el significado de las fórmulas químicas y de las ecuaciones químicas. De hacer énfasis en la importancia de la utilización del concepto de mol.

A pesar de que aparecen nuevas sustancias, la masa se conserva en una reacción química.

\section{Comentario}

Con relación a la frase del segundo profesor, es claro que no es sino pasando primero por la naturaleza nanoscópica de la RQ y la identificación de elementos en las fórmulas de las sustancias iniciales y finales que se llega a la conclusión de que se da la conservación elemental en una reacción química.

Solsona e Izquierdo (1998) nos indican que la conservación de los elementos químicos es un concepto francamente difícil de asimilar por los estudiantes.

De aquí que Griffiths y Preston (1992) señalen que "los educadores de la Quími- ca generalmente están de acuerdo en que la comprensión de los conceptos átomo y molécula son fundamentales para el aprendizaje de la Química".

Es consecuente, pues, la insistencia del segundo profesor que menciona la necesidad de que los alumnos "comprendan el significado de las fórmulas químicas y de las ecuaciones químicas", es decir, que los alumnos transiten de la comprensión macroscópica de una RQ a su comprensión nanoscópica, con claridad acerca de la verdadera transformación de la estructura molecular.

II. En cuanto a 'representación de reacciones', dos de los profesores nos citan lo siguiente en la pregunta 4 , las dificultades y limitaciones relacionadas con la enseñanza de esta idea: Pasar al mundo de lo simbólico requiere de aclaraciones constantes por parte del profesor. Todo se trata de presentar un modelo representacional que resulte sintético y valioso para el entendimiento de la Química.

La forma simbólica de representar una reacción química es por medio de una ecuación química.

\section{Comentario}

Con relación a la representación de reacciones químicas mediante símbolos, Gabel (1999) cita el triángulo de Johnstone (véase Figura 1): "La materia que es observada y puede ser estudiada al nivel macroscópico, puede también ser descrita al nivel nanoscópico y, en ocasiones, al hacerlo parece que las explicaciones son más definitivas. Para complicar más las cosas, los químicos pueden representar ambos niveles, el macroscópico y el nanoscópico, mediante el empleo de símbolos químicos, formulas químicas y 
ecuaciones químicas”. Es una destreza muy particular del profesor de Química ir acostumbrando a los estudiantes a este cambio de foco de la Química, que lo lleva, de manera natural, de uno de los vértices del triángulo de Johnstone al otro.

Figura 1. El triángulo de Johnstone. Estos tres niveles debe abarcar la enseñanza de la química. Hay que aclarar que Johnstone empleó 'submicroscópico' en lugar de 'nanoscópico'.

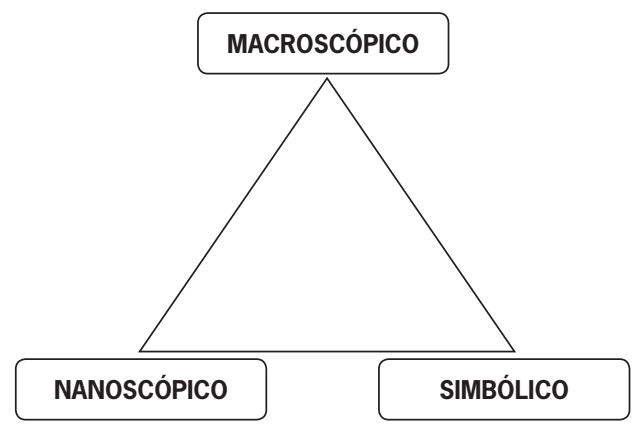

III. Con relación al tema 'relaciones cuantitativas de la reacción química y balanceo'

a) Se presentan a continuación los siguientes objetivos de aprendizaje de dos de los profesores (pregunta 1):

Lo que aquí se intenta es que los estudiantes sean capaces de realizar los cálculos estequiométricos involucrados en una reacción química. Para comprender este tema es necesario que cuenten con el concepto de cantidad de sustancia y su unidad, la mol, y que tengan destreza en el manejo del concepto de proporcionalidad.

Establecer la proporción en que intervienen los reactivos para predecir la cantidad de productos que se obtendrán a través de cálculos estequiométricos sencillos.

\section{Comentario}

El concepto cantidad de sustancia y su unidad, la mol, ha sido uno ampliamente debatido en la literatura educativa (Dierks, 1981; Strömdahl et ál., 1994; Furió et ál., 2000), sobre todo el poco uso que se da del primero en los libros de texto de Química, por lo cual resulta gratificante la frase del primer profesor.

Para llevar a cabo la cuenta de las masas involucradas en una reacción de todos los compuestos participantes, es muy útil emplear una tabla de reacción (Watkins, 2003), que es lo que parece recomendar el segundo profesor con su frase.

b) Para la idea 'balanceo de ecuaciones', presentamos las frases de tres profesores con respecto a los conocimientos acerca del pensamiento de los estudiantes que influyen en su enseñanza de esta idea (pregunta 5):

Los profesores con frecuencia olvidamos que gran parte del lenguaje que usamos para describir fenómenos químicos no es obvio para los estudiantes. Se debe tener en cuenta que las fórmulas, ecuaciones y otras representaciones simbólicas usadas en química no son familiares a los estudiantes y que ellos están aprendiendo un nuevo lenguaje. En una ecuación química hay una serie de ítems de las que los estudiantes deben aprender su significado: fórmulas, símbolos como: $\Delta, \downarrow, \uparrow \leftrightarrow \rightarrow$, s, l, g. Los subíndices y los coeficientes son frecuentemente confundidos.

Desconocen el lenguaje químico, por lo cual no saben escribir las fórmulas, no aplican el principio de la conservación de la masa, por lo cual no balancean las ecuaciones químicas y modifican los subíndices 
de las fórmulas para balancear las ecuaciones.

Los alumnos deben superar la dificultad que tienen en distinguir que en una fórmula química los subíndices indican el número de átomos de cada elemento presente y el coeficiente indica el número de fórmulas que hay que considerar y que afecta a todos los elementos presentes en el compuesto.

\section{Comentario}

La alerta del primer profesor acerca de lo novedosa que es toda la simbología química para los alumnos ha de tomarse en consideración para el desarrollo del CPC de los profesores novatos.

Añade complejidad a la Química el uso frecuente de símbolos matemáticos, fórmulas y ecuaciones para expresar relaciones a los niveles macroscópico y nanoscópico (Gabel, 1999).

Con relación a la segunda y tercera frases, ha sido advertida desde hace tiempo en la literatura (Paoloni, 1979; Yarroch, 1985) la confusión que se da entre los alumnos de los subíndices y los coeficientes estequiométricos en una reacción.

IV. Para la 'formación de nuevas sustancias', dos de los profesores nos expresan las siguientes estrategias a utilizar (pregunta 7):

Conviene al empezar el tema hablar de lo vano que resulta diferenciar los cambios físicos de los cambios químicos, sobre todo si se menciona que los cambios químicos involucran la transformación sustancial del ambiente submicroscópico, porque una disolución o un cambio alotrópico implican sin duda una transformación notable del ambiente submicros- cópico, siendo que son clasificados normalmente como cambios físicos. De forma similar, otros cambios químicos como la desnaturalización de la proteína del huevo al hervirlo, no conducen a un cambio en el orden de enlace de los aminoácidos, sino únicamente a la presencia o ausencia de estructura terciaria de las proteínas involucradas.

En el enfoque tradicional no se da tiempo ni espacio para consolidar el aprendizaje de un concepto que es necesario para otros subsecuentes. Se utilizan pocos ejemplos demostrativos o de imágenes que ayuden a los alumnos a reconocer que se ha formado una nueva sustancia para que traten de explicar que ese cambio ocurre a nivel submicroscópico entre los átomos. Se pueden dar problemas prácticos para reconocer la identidad de diferentes muestras de sustancias a través de realizar pruebas con diferentes reactivos y comparar resultados.

\section{Comentario}

El primer profesor apunta un problema fundamental en la enseñanza de los cambios físicos como algo diferenciado de los cambios químicos, un debate que tiene ya muchos años (Gensler, 1970; Strong, 1970) y que escogimos para documentar en forma de un inventario (ver Apéndice 2).

Con relación a la frase del segundo profesor, Bond-Robinson (2005), quien presenta un trabajo sobre CPC en el laboratorio químico, nos indica que "entender el significado químico de una reacción es correlacionar el cambio químico visible con un modelo mental de átomos, iones y moléculas reaccionando en el mundo nanoscópico." Este paso de 
la descripción macro a la nano, relativa a las partículas, resulta ser el paso crucial en la enseñanza del concepto de RQ (de Vos y Verdonk, 1985; 1986; 1987).

V. Y por último para la idea 'tipos de reacciones', dos de los profesores nos citan en la pregunta de los procedimientos de enseñanza:

El laboratorio es un magnífico aliado para demostrar y comprender esta idea. Se propone la realización de valoraciones sencillas ácido-base, haciendo énfasis en el funcionamiento de diferentes indicadores y la ilustración de las reacciones redox.

Realizar experimentalmente, y de preferencia con técnicas de microescala, una variedad de cambios químicos. Mostrar reacciones que involucren cambios energéticos y ayudarles a reconocer que se forman nuevas sustancias (cambio de color, de olor, formación de gases, de precipitados, etc.) y extender este razonamiento a otras reacciones para establecer posibles criterios que permitan clasificar las reacciones.

Con relación al énfasis dado en estas respuestas al trabajo práctico, conviene recordar lo que nos indican Hofstein y Lunetta (2004) para su desarrollo ulterior durante este siglo:

Al laboratorio se le ha dado un papel central y distintivo en la Educación en Ciencias, y los educadores de la ciencia han sugerido los ricos beneficios del aprendizaje acumulado al emplear actividades de laboratorio [...] Vivimos en una era de cambios dramáticos en los recursos de nuevas tecnologías y nuevos estándares de la Educación en Ciencias en los que el aprendizaje por indagación (inquiry) ha adquirido un renovado estatus central.
Cada ReCo permite apreciar una clara línea de trabajo de cada uno de los profesores. A continuación presentamos la característica que distingue a cada uno de ellos, con una frase textual que la revela:

1. El profesor 1 concibe a la Química más como una ciencia donde el término 'sustancia' es el fundamental, que otra en la que el término 'reacción química' lo sea. Se trata de un profesor con una fuerte componente teórica, como revela la siguiente frase de su CoRe:

La descripción adecuada de estas interacciones requiere del complejo y bello aparato de la mecánica cuántica, aunque las interacciones entre partículas también se pueden describir satisfactoriamente con modelos eléctricos clásicos como dipolos eléctricos y cargas puntuales.

Sin embargo, nos aclara:

Los modelos que describen estas interacciones son de una complejidad matemática muy superior a la que poseen los alumnos (y los profesores) de este nivel. Sería un error pretender sustentar esta idea con el rigor matemático propio de los modelos. Sin embargo, sí es factible presentar los conceptos centrales.

2. Los profesores 2 y 3 tienen en su habitual expresión en el aula una fuerte componente experimental. Hemos extraído un párrafo de cada CoRe: Realizar actividades experimentales en que se varíe la proporción de uno de reactivos y el otro se mantenga constante (técnica de las variaciones múltiples). Pueden utilizarse reacciones de precipitación en que los alumnos puedan separar fácilmente el producto formado y determinar su masa. Presentar los resultados en 
gráficos para establecer la proporción en que varía cada uno y detectar los posibles errores experimentales en la determinación de las masas. Ésta es, sin duda, una forma fácil de obtener las fórmulas de los compuestos de forma cuantitativa.

El laboratorio es un magnífico aliado. Se llevan a cabo experimentos del tipo de ciclos de reacciones, que demuestran la conservación de la materia y que involucran la práctica de la nomenclatura y de los cálculos estequiométricos. Pueden también llevarse a cabo reacciones endotérmicas y exotérmicas. O bien, realizar valoraciones sencillas ácido-base, enfatizando el funcionamiento de diferentes indicadores, o ilustrar las reacciones redox, demostraciones del Principio de Le Chatelier y la demostración de la reversibilidad de algunas reacciones.

3. El profesor 4 es un químico teórico que pone énfasis en algunas concepciones alternativas de los estudiantes (Kind, 2004):

La palabra 'sustancia' tiene una concepción cotidiana no tan restrictiva como la 'sustancia' de la Química, lo cual dificulta su aprendizaje.”

El proceso de ‘aparición' y ‘desaparición' de sustancias no es comprendido cabalmente por los alumnos, pues interfiere con su concepción acerca de la conservación de la materia.

Algunos alumnos tienen problema en identificar las proporciones atómicas en la escritura de fórmulas, a base de subíndices y paréntesis.

4. El profesor 5 utiliza el Triángulo de Johnstone para clasificar sus ideas centrales, al que añade una cuarta componente a la que denomina 'dimensión cuantitativa':
- Dimensión macroscópica

- Formación de nuevas sustancias.

- Conservación de la masa.

- ¿Cómo se sabe que se ha llevado a cabo una reacción química?

- Dimensión nanoscópica

- Discontinuidad de la materia.

- Conservación de los átomos.

- Conceptos de sustancia, átomo y molécula.

- Dimensión simbólica

- Representación de reacciones.

- Balanceo de ecuaciones.

- Dimensión cuantitativa

- El concepto de cantidad de sustancia y su unidad el mol.

- Relaciones en masa y en cantidad de sustancia, reactivo limitante, rendimiento, pureza de los reactivos, etc.

Algunas cuestiones que resaltan entre sus respuestas al cuestionario de la

\section{ReCo fueron:}

Para que los estudiantes comprendan las reacciones químicas, deben manejar con soltura la noción de discontinuidad de la materia. La materia está formada por partículas: átomos que se combinan formando moléculas. Por otra parte, deben comprender la conservación de los átomos de cada elemento durante el transcurso de la reacción. Una gran parte de los estudiantes no tiene estos conceptos y considera que la materia es continua, por lo que no puede comprender a cabalidad lo que es una reacción química.

Considero que el principal problema que tienen los estudiantes en los cálculos estequiométricos involucrados en las reacciones químicas, es que no manejan la proporcionalidad y se les presenta muy abstracto el concepto de cantidad de sustancia. 
En efecto, con relación al manejo de proporciones se ha mencionado que pocos estudiantes han alcanzado el estadio piagetiano de las operaciones formales, así que no pueden comprender el concepto de mol (Herron, 1975).

En lo relativo a los inventarios recabados, el profesor 4 nos permitió grabar su clase para elaborar el inventario que resumimos en el Apéndice 2. Éste revela la problemática que existe alrededor de la definición de los conceptos 'reacción química' y 'sustancia'. En tanto, la profesora 5 nos permitió ver las reacciones que desarrolla con sus alumnos, a partir de lo cual realizamos el inventario que resumimos en el Apéndice 1. Es de resaltar la claridad de esta profesora en la necesidad de pasar de los aspectos macroscópicos a los nanoscópicos.

\section{Conclusiones}

La metodología propuesta por Loughran, Mulhall y Berry (2004) permitió una eficaz documentación del CPC de los profesores. La disposición y arreglo de los datos facilitó el análisis de los mismos. Resulta crucial hacer énfasis en que la ReCo es tanto una herramienta de investigación para acceder al entendimiento del contenido por parte de los profesores involucrados, como una manera de representar este conocimiento.

En las ReCos obtenidas, los profesores identifican claramente las ideas centrales asociadas con la RQ y los objetivos de su enseñanza, reconocen las probables dificultades conceptuales de sus estudiantes y plasman las estrategias más empleadas para abordar satisfactoriamente la clase y su evaluación. En la comparación de las ideas centrales de profesores australianos reportadas por Mulhall, Berry y Loughran (2003), tres de ellas fueron informadas por al menos tres de nuestros profesores y una cuarta por dos de ellos.

Por lo detectado en las ReCos, quizás el mayor problema que presenta la enseñanza de este tema es que en ocasiones, para muchos alumnos, la aparición de nuevas sustancias no es visible; así mismo, se les dificulta comprender que a pesar de que se conserve la masa en una reacción aparezcan nuevas sustancias, ya que, argumentan, esto actúa en contra del 'principio de conservación'. Se recomienda que el estudiante observe y analice varias RQ hasta que saque sus propias conclusiones acerca de lo que una RQ representa y después pasar a una representación nanoscópica de la misma, de tal manera que asimile que se conservan los átomos de los elementos químicos.

Resumiendo, los aspectos que los profesores entrevistados insisten como importantes para la enseñanza de este tema son:

- Conocer las concepciones alternativas de los estudiantes.

- La forma de comprobar que existe conservación de la masa en una reacción química es llevándola a cabo experimentalmente en un recipiente cerrado sobre una balanza.

- El balance de elementos es algo fundamental para comprender la porción cuantitativa de las reacciones y la conservación de la materia.

- Lo fundamental de la idea de 'R.

- Representación de reacciones' es que toca las tres puntas del Triángulo de Jonhstone.

- El uso del concepto de cantidad de sustancia resulta prioritario para 
trabajar adecuadamente la fase cuantitativa del tema.

- Hay que poner atención especial con relación a los aspectos del lenguaje químico.

- Resulta vano hablar de cambios físicos y cambios químicos.

- Primero hablar del concepto de sustancia, después se puede entrar a la $\mathrm{RQ}$, definiéndola como un proceso en el que unas sustancias se transforman en otras.

- Es muy importante la enseñanza experimental para la idea central de tipos de reacciones.

- Hay que ver antes del tema de reacción química el de estructura corpuscular de la materia.

Como característica importante de los inventarios elaborados por los profesores (que se encuentran en los dos apéndices) se encontró que reúnen preguntas o actividades que apoyan a los estudiantes a explorar conceptos centrales.

La perspectiva que emerge de esta investigación es emplear tanto las ReCos como los inventarios obtenidos para que sean discutidos por profesores en formación en talleres de trabajo. A pesar de la insistencia de que el CPC se adquiere mayoritariamente como una expresión de la propia docencia, el emplear estas muestras de ejemplos de profesores distinguidos en los talleres formativos resultará, seguramente, de utilidad porque reduce la novedad y la sorpresa, ya que le da mayor capacidad de respuesta al profesor en formación ante posibles situaciones que lo puedan tomar inadvertido, generándose un círculo virtuoso en el que se incrementa su confianza.

\section{Apéndice 1}

\section{Un inventario² de una profesora}

Este inventario presenta una clase práctica para explicar las nociones sobre la RQ. La maestra presenta su forma de trabajo con el grupo, las demostraciones experimentales que utiliza y los rasgos generales de análisis y las reflexiones que hace en clase.

Como actividad inicial y para detectar algunas de las ideas que tienen los estudiantes sobre lo que es una reacción química, se forman equipos de cuatro personas y se les entregan dos bolsas de polietileno con cierre hermético. Cada bolsa contiene un polvo blanco. Se pide a los estudiantes que mezclen en una sola bolsa las dos sustancias y que le añadan, con una pipeta de $10 \mathrm{~mL}$, la disolución de una sustancia líquida (que es rojo de fenol). Se les recomienda que tengan la precaución de cerrar perfectamente su bolsa de plástico y se les pide que escriban todas sus observaciones. Los reactivos son bicarbonato de sodio y cloruro de calcio. Lo que se observa es el desprendimiento de un gas, el cual 'infla la bolsa', se observa también un cambio de coloración del líquido agregado y un considerable aumento en la temperatura.

Después de esta actividad, continúa con una lluvia de ideas sobre los fenómenos que los estudiantes han observado y que pueden considerar que se trata de un cambio químico. Para los alumnos resultan familiares fenómenos como

2 El término inventario fue propuesto por Raviolo y Garritz (2005) a partir del de "Repertorios de experiencia profesional y pedagógica" (PaP-eRs son sus siglas en inglés), originalmente planteados por Loughran et ál. (2001). 
la combustión, pues ésta ha formado parte de los juegos de muchos de ellos desde su niñez. También mencionan la explosión de petardos, bengalas e incluso recuerdan cuando mezclaban azufre, carbón y pastillas de clorato para preparar sus propios explosivos.

Después se procede a realizar una serie de reacciones químicas en donde se note que hay desprendimiento de gases, cambio de color, formación de precipitados, etc. (ver la figura 2) y se enfoca la discusión en el sentido de que se perciban las propiedades tanto de las sustancias que reaccionan como de las sustancias que se forman. Lo importante es que los estudiantes vean que se forman nuevas sustancias que antes no existían.
Se hace énfasis en que cuando ocurre una reacción química las propiedades de las sustancias obtenidas son muy diferentes a las de los reactivos. El sodio es un metal muy activo que al combinarse con el cloro, que es un gas tóxico amarillo-verdoso, produce el cloruro de sodio, sólido cristalino, con el que acentuamos el sabor de nuestros alimentos.

Aquí también se hace notar que existen algunas reacciones en las que, cuando tienen lugar, no se observa cambio alguno, como es el caso de las reacciones ácido-base en disolución. Para notar el cambio químico se tiene que hacer uso de indicadores o detectar cambios de la temperatura.

Figura 2. Algunas de las reacciones vistosas que conviene presentar a nuestros estudiantes
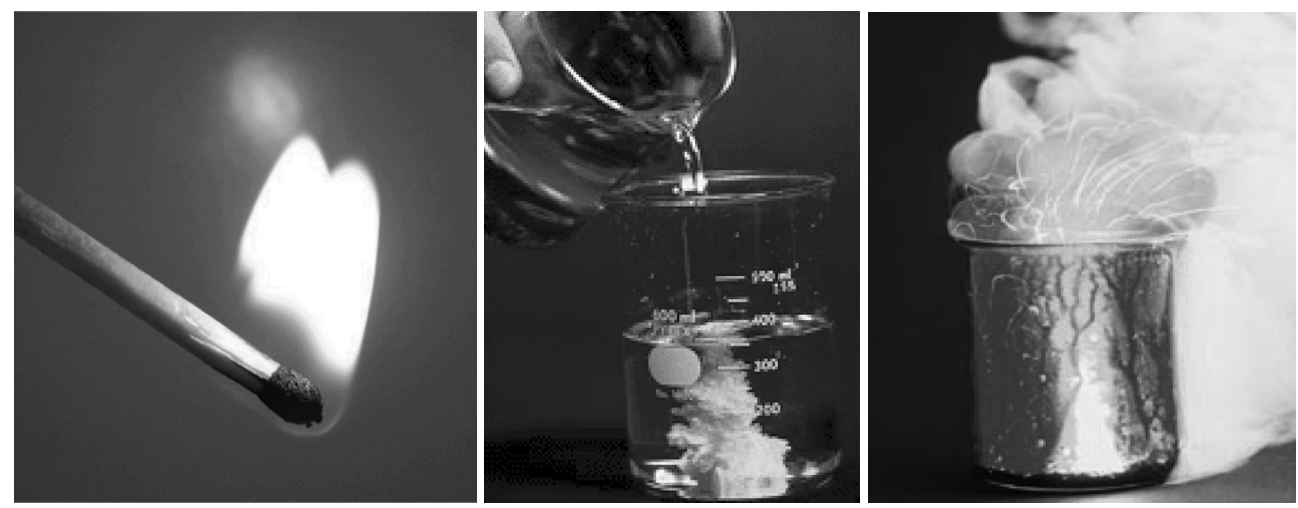

La profesora puso énfasis también en el empleo de las Tecnologías de la Información y la Comunicación, TICs, para presentar a los alumnos imágenes de reacciones químicas a nivel nanoscópico, como ha sido resaltado recientemente por Ardac y Akaygun (2005).

Estas actividades pueden realizarse aproximadamente en dos horas.

\section{Apéndice 2}

\section{Un inventario de un profesor}

Este inventario presenta una clase teórica sobre lo que es 'la Química' abordada por aprendizaje cooperativo, en grupos de tres estudiantes, en el cual a partir de disertaciones teóricas se analizan los conceptos sustancia, reacción química 
y Química. La pregunta central, para empezar, es:

"QUÍMICA: ¿Tiene que ver con sustancias o con procesos?

En esta clase vamos a plantear la dicotomía existente para la Qímica, que está presente en las siguientes preguntas:

- ¿Es la Química una ciencia dedicada al estudio de cosas o al de procesos? ¿Es una ciencia que trata acerca de sustancias o acerca de reacciones químicas?

- ¿Se define una reacción química por la transformación de ciertas sustancias en otras o las sustancias son definidas por sus reacciones químicas características?"

Por ejemplo, Steven Weininger (2000) apunta que a pesar de que los procesos químicos son el centro de gran parte de la investigación química, el aparato conceptual para describir estos procesos es sorprendentemente pobre comparado con el que existe para describir estructuras.

Parte el profesor de una primera definición de Química (la de Ronald Stanley Nyholm, un notable químico canadiense):

La Química es el estudio integrado de

la preparación, propiedades, estruc-

tura y reacciones de los elementos

y sus compuestos, así como de los

sistemas que forman.

Recuerda entonces la definición de ‘sustancia química' de la IUPAC, para incluirla en una nueva definición de 'Química' (nótese que no aparece caracterizada una sustancia por sus propiedades químicas, sino sólo por las entidades elementales de las que está formada y por sus propiedades físicas):
Materia de composición constante mejor caracterizada por las entidades de las que está compuesta (moléculas, unidades fórmula o átomos). Las propiedades físicas tales como la densidad, el índice de refracción, la conductividad eléctrica, el punto de fusión, etc., caracterizan a la sustancia química.

A partir de la primera definición, añadiendo nuevos elementos y la reunión del concepto de sustancia, el profesor y sus alumnos arriban a una segunda definición.

La Química es el estudio integral y controlado de las sustancias, su modo de obtención, estructura interna, propiedades, caracterización, transformaciones estructurales y cambios de energía presentes en éstas.

La 'reacción química' está escondida en el concepto 'transformaciones estructurales' y el de 'sustancia' ha reemplazado al de 'los elementos y sus compuestos', convirtiéndose en el término más importante de la definición. No cabe duda, sin embargo, que una definición como ésta hace resaltar el estudio de las sustancias como algo fundamental en Química y no tanto el estudio de los procesos químicos. Pero, ¿existen de verdad procesos químicos realmente diferenciados de los procesos físicos?

Dice entonces el profesor que "siempre se falla en el intento de definir un universo de estudio de la Física perfectamente ajeno al de la Química". No existe una frontera claramente definida entre ambas, lo que afortunadamente habla acerca de la unidad de la ciencia. Desde 1970 se adoptan tanto la actitud de defensa como la de ataque con relación a la enseñanza 
de una distinción entre 'cambios físicos' y 'cambios químicos' en dos artículos de ese año en el Journal of Chemical Education (Gensler, 1970; Strong, 1970).

Posteriormente el profesor va pasando por una multitud de conceptos tales como 'entidad elemental', 'reacción química', 'especie química', 'cambio físico y cambio químico', presentando sus definiciones de la IUPAC entre las discusiones, para concluir que: "no hay sino una frontera nebulosa entre los cambios físicos y los químicos". Por ello, se dice que hacer esa distinción en el aula no es una ayuda pedagógica ni tampoco significativa desde el punto de vista científico (Borsese y Esteban, 1998). Se trata, en última instancia, de modelos extremos sobre el comportamiento de la materia en los procesos naturales. Y en Ciencia es elemental no confundir los modelos con la realidad. "Quizá podamos encontrar casos reales de transformaciones de la materia que se adapten cercanamente a alguna definición arbitraria o convencional de modelo de cambio físico o a una de cambio químico, pero nada más".

Además, hemos tenido que introducir inevitablemente en el análisis y discernimiento de uno $\mathrm{u}$ otro tipo de procesos la noción nanoscópica de la estructura interna de la materia. Así, hemos de resignarnos a perder la posibilidad de definir el concepto desde un punto de vista puramente observacional, para entrar en las entrañas del nanocosmos, tan abstracto, ajeno y distante al alumno.

El artículo de Palmer y Treagust (1996) analiza los criterios que se han dado en los libros de texto para diferenciar cambios físicos de químicos durante los últimos ciento setenta años, y plantea cuatro hipótesis acerca de la longevidad de este tema en los libros:

El concepto es un remanente de la teoría aristotélica de la materia, retenido por el conservadurismo natural de los científicos.

La distinción entre cambio físico y químico en los libros es un artilugio pedagógico, que ayuda a los estudiantes a entender los conceptos relacionados.

El concepto puede ser ilustrado por un conjunto de experimentos excitantes e interesantes que le satisfacen a aquellos profesores que se ven a sí mismos orientados hacia el trabajo práctico.

El concepto es un ardid empleado por los químicos para definir la frontera entre Química y Física, con ventaja para la Química, de tal forma que los jóvenes tiendan a seleccionar esta disciplina como objeto de estudio, en lugar de la Física.

Finalmente, el profesor plantea que hay quien toma un punto de vista intermedio ante preguntas como las que inician este inventario y llega a la conclusión de que dicha dicotomía es un problema no resuelto:

Shummer (2004) ha argumentado hacia un enfoque integrador de la dicotomía mencionada, que combine conceptualmente sustancias y procesos químicos en una red de relaciones dinámicas, de tal forma que sustancias y reactividades se definan mutuamente una a la otra, tanto al nivel teórico como en el experimental. $\boldsymbol{\Delta}$ 


\section{Bibliografía}

Ardac, D. y Akaygun, S. (2005). Using static and dynamic visuals to represent chemical change at molecular level. International Journal of Science Education, 27, 11, 1269-1298.

Barnett, J. y Hodson, D. (2001). Pedagogical Context Knowledge: Toward a fuller understanding of what good science teachers know. Science Education, 85, 426-453.

Bond-Robinson, J. (2005). Identifying pedagogical content knowledge (PCK) in the chemistry laboratory. Chemistry Education Research and Practice, 6, 2, 83-103.

Borsese, A. y Esteban, S. (1998). Los cambios de la materia, ¿deben presentarse diferenciados como químicos y físicos? Alambique. Didáctica de las Ciencias Experimentales, 17, 85-92.

De Jong, O., Veal, W. R. y Van Driel, J. H. (2002). Exploring chemistry teachers' knowledge base. En J. K. Gilbert et ál. (Eds.), Chemical Education: Towards Research-based Practice. The Netherlands: Kluwer Academic Publishers, 369-390.

De Vos, W. y Verdonk, A. H. (1985) A new road to reactions. Part 1. Journal of Chemical Education, 62, 3, 238-240.

De Vos, W. y Verdonk, A. H. (1985) A new road to reactions. Part 2. Journal of Chemical Education, 62, 8, 648-649.

De Vos, W. y Verdonk, A. H. (1986) Part 3 . Teaching the heat of reactions. Journal of Chemical Education, 63 , 11, 972-974.

De Vos, W. y Verdonk, A. H. (1987) Part 4. The substance and its molecules, Journal of Chemical Education, 64, 8, 692-694.

De Vos, W. y Verdonk, A. H. (1987) Part 5. The elements and its atoms, Journal of Chemical Education, 64, 12, 10101013.
Dierks, W. (1981). Teaching the Mole. European Journal of Science Education, 3, 145-158.

Furió, C., Azcona, R. Guisasola, J. \& Ratcliffe, M. (2000). Difficulties in teaching the concepts 'amount of substance' and 'mole'. International Journal of Science Education, 22(12), 1285-1304.

Gabel, D. (1999). Improving teaching and learning through chemistry education research: A look to the future. Journal of Chemical Education, 76, $4,548-554$.

Garritz, A. y Trinidad-Velasco, R. (2004). El conocimiento pedagógico del contenido, Educación Química, 15, 2, 98-101.

Garritz, A. y Trinidad-Velasco, R. (2006). El conocimiento pedagógico de la estructura corpuscular de la materia. Educación Química, 17, núm. extraord., 236-263.

Garritz, A. y Valdéz, R. (2007). El Conocimiento Pedagógico del Contenido (CPC) ¿Cuál es la diferencia entre un buen y un mal maestro? En Iniciación a la cultura científica. La formación de maestros. Madrid: Editorial Antonio Machado, colección "aprendizaje".

Gensler, W. J. (1970). Physical versus chemical change. Journal of Chemical Education, 47, 2, 154-155.

Herron, J. D. (1975). Piaget for chemists. Journal of Chemical Education, 52, 146-150.

Hofstein, A. y Lunetta, V, N. (2004). The laboratory in science education: Foundation for the 21st century. Science Education, 88, 28-54.

Kind, V. (2004). Más Allá de las Apariencias. Ideas Previas de los Estudiantes sobre Conceptos Básicos de Química. México: Aula XXI Santillana, Univer- 
sidad Nacional Autónoma de México, UNAM.

Loughran, J., Milroy, P., Gunstone, R., Berry, A. y Mulhall, P. (2001). Documenting science teachers' pedagogical Content Knowledge Trough PaP-eRs. Research in Science Education, 31, 289-307.

Loughran, J., Mulhall, P. y Berry, A. (2004). In search of Pedagogical Content Knowledge in science: Developing ways of articulating and documenting professional practice. Journal of Research in Science Teaching, 41, 4, 370-391.

Mulhall, P., Berry, A. y Loughran, J. (2003). Frameworks for representing science teachers' pedagogical content knowledge, En Asia Pacific Forum on Science Learning and Teaching. 4, 2, 2. Acceso en Noviembre 5, 2006, de la URL http://www.ied.edu.hk/ apfslt/v4_issue2/mulhall/index. htm\#contents.

Palmer, W. y Treagust, D. F. (1996). Physical and chemical changes in textbooks: an initial view, Research in Science Education, 26, 1, 129-140.

Paoloni, L. (1979). Towards a culturebased approach to chemical education in secondary schools: the role of chemical formulae in the teaching of chemistry. European Journal of Science Education, 1, 4, 365-377.

Pozo, J. I. y Gómez Crespo, M. A. (1997). Aprender y enseñar Ciencia. Madrid: Morata.

Pozo, J. I. y Gómez Crespo, M. A. (1997). ¿Qué es lo que hace difícil la comprensión de la ciencia? Algunas explicaciones y propuestas para la enseñanza. En Del Carmen, L. (coord.), La enseñanza y el aprendizaje de las ciencias de la naturaleza en la educación secundaria, Cuadernos de formación del profesorado. (2 Edición). Barcelona: ICE/Horsori, 73-105.
Raviolo, A. y Garritz, A. (2005). Decálogos e inventarios. Educación Química, 16, Núm. extraord., 106-110.

Reyes-C., F. y Garritz, A. (2006). Conocimiento pedagógico del concepto de 'reacción química' en profesores universitarios mexicanos. Revista Mexicana de Investigación Educativa, $11,3,1175-1205$.

Shulman, L. S. (1986). Those who understand: Knowledge growth in teaching, Educational Researcher, 15, 2, 4-14.

Shulman, L. S. (1987) Knowledge and teaching: foundations of the new reform. Harvard Educational Review, $57,1-22$.

Schummer, J. (2004). Editorial: substances versus reactions. HYLE, International Journal for the Philosophy of Chemistry, 10, 1, 3-4.

Strömdahl, H., Tulberg, A. \& Lybeck, L. (1994). The qualitatively different conceptions of 1 mole. International Journal of Science Education, 16(1), 17-26.

Strong, L. E. (1970). Differentiating physical and chemical changes. Journal of Chemical Education, 47, 10, 689690.

Watkins, S. F. (2003). Applying the reaction table method for chemical reaction problems (stoichiometry and equilibrium). Journal of Chemical Education, 80, 6, 658-661.

Weininger, S. J. (2000). Butlerov's vision. The timeless, the transient, and the representation of chemical structure. En N. Bhushan y S. Rosenfeld (Eds.) Of Minds and Molecules. New York: Oxford University Press, 143-161.

Yarroch, W. L. (1985). Student understanding of chemical equation balancing. Journal of Research in Science Teaching, 22, 449-453. 